\title{
CORRESPONDENCES AND THE THEORY OF GROUPS*
}

\author{
BY \\ JOSEPH EDMUND WRIGHT
}

The object of this paper is to bring together several points connected with the general theory of correspondences and continuous groups, and to apply them to the theory of screws. Although the several results are in general not new, it seems of interest to give the accompanying presentation of the subject, as it furnishes an excellent example of the way in which the theory of continuous groups underlies the whole theory of correspondences. $\dagger$

The first section is devoted to general theory. Use is made of the theorem of LIE $\ddagger$ that if we have a continuous group in $n$ variables together with an invariant equation system involving $m$ parameters, then a 'group of the parameters' exists which is isomorphic with the given group, and it is pointed out that this theorem is fundamental in all correspondences. $\S$ The correspondence established is that between a $P_{n}$ and a $P_{n}$. Contact transformation is the particular case when $m=n$.

The screw geometry is developed from the projective group in three dimensions together with the system of equations which define a general straight line. The general theory leads at once to two important results in connection with the theory of groups :

1) The general continuous conformal group in four dimensions is simply isomorphic with the general projective group in three.

2) Both these groups are simply isomorphic with the continuous projective group in five dimensions which leaves a given quadric invariant.

There follows an immediate generalization of part of the second theorem. We have in fact the following:

3) The general conformal group in space of $n$ dimensions is simply isomorphic with the projective group in spiace of $n+1$ dimensions which preserves a given quadric.

These three results are due to KLEIN. $\|$ Some slight differences appear

* Presented to the Society December 29, 1905. Receiver for publication January 9, 1906.

† See Klein, Erlangen Programme, 1872 ; Bulletin of the American Mathematical Society, vol. 2 (1893), pp. 215 sqq.

† See Continuirlichen Gruppen, pp. 549, 718.

\& KLEIN, loc. cit.

I See in particular Höhere Grometrie, vol. 1, p. 487 sqq. 
because we are concerned merely with continuous groups; for example, our conformal group does not include inversions. The theory leads naturally to KLEIN's * correspondence between linear complexes and hyperspheres, and the properties of that correspondence are developed by a priori reasoning. The sphere-straight line correspondence of $\mathrm{LIE}_{\mathrm{E}}$ also appears in the natural course of development. The major part of the remainder of the paper is concerned with the correspondence shown to exist between points in space of five dimensions and screws. With respect to particular results we may mention that reciprocal screws become conjugate points with respect to the fundamental quadric, and that the process of finding the resultant of any number of wrenches on given screws is shown to be equivalent to the process of finding the mass centre of given masses at given points in the five dinensional space.

The following notation is used throughout: $P_{m}$ denotes a linear $m$ dimensional manifold in ordinary space of any dimensions; $S_{m}$ denotes a hypersphere of $m$ dimensions in ordinary space of any dimensions. A 'quadric' is a locus satisfying an equation of the second degree in any space.

Let

\section{$\S 1$.}

$$
x_{i}^{\prime}=f_{i}\left(x_{1}, \cdots, x_{n}, a_{1}, \cdots, a_{r}\right) \quad(i=1, \cdots, n),
$$

be the finite equations of a continuous group in the variables $x$, and let there be any equation system

$$
\phi_{s}\left(x_{1}, \cdots, x_{n}, y_{1}, \cdots, y_{n}\right)=0 \quad(8=1, \cdots, p),
$$

such thai, for all transformations of the group, values $y_{1}^{\prime}, \cdots, y_{m}^{\prime}$ independent of $x$ exist which make

$$
\phi_{s}\left(x_{1}^{\prime}, \cdots, x_{n}^{\prime}, y_{1}^{\prime}, \cdots, y_{m}^{\prime}\right)=0 \quad(s=1, \cdots, p),
$$

provided the $\phi$ 's in the unaccented variables vanish. The general theory of groups shows that the transformations for the $y$ 's form a group which is isomorphic with the original one. This group need not necessarily be of the same order as the original one. Consider for example the general linear group

$$
x_{i}=\sum_{j=1}^{n} \alpha_{i j} x_{j} \quad(i=1, \cdots, n),
$$

in conjunction with the equation system

$$
x_{i}=y_{i} x_{n} \quad(i=1, \cdots, n-1),
$$

đKLein, Mathematische Annalen, vol. 5 (1872) p. 257 ; see also Grace, Transactions Cambridge Philosophical Society; vol. 16 (1898), p. 153. 
The group for the $x$ 's is of order $n^{2}$, whereas the group for the $y$ 's, the general projective group in $(n-1)$ dimensions, is only of order $n^{2}-1$.

Let $X f f$ denote any infinitesimal operator of the $x$ group, and $Y f$ the corresponding operator of the $y$ group, then the structure constants are the same for the two cases, but there may be linear relations between the operators of the $y$ group. In any case the equations $\phi=0$ form an invariant equation system for the group whose operators are $(X+Y) f$, and this group is isomorphic with both the $x$ and $y$ groups.

The general condition for simple isomorphism is readily obtained. Suppose that the $y$ group is of order $r-h$, then there must exist $h$ infinitesimal transformations of the $x$ group which transform the system

into the system

$$
\phi_{s}\left(x_{1}, \cdots, x_{n}, y_{1}, \cdots, y_{m}\right)=0 \quad(s=1, \cdots, p),
$$

$$
\phi_{s}\left(x_{1}^{\prime}, \cdots, x_{n}^{\prime}, y_{1}, \cdots, y_{m}\right)=0 \text {. }
$$

There must thus exist a subgroup of the $x$ group for which the equations $\phi=0$ form an invariant system, for all values of the $y$ 's. For instance, in the case considered, $x_{i}^{\prime}=\lambda x_{i}$ is such a subgroup of the general linear group.

Now consider any manifold of $n$ dimensions and let any element in it be determined by $x_{1}, \ldots, x_{n}$. Consider also any manifold of $m$ dimensions in which an element is determined by $y_{1}, \cdots, y_{m}$. Then we say that a correspondence exists between the two manifolds such that to an element of the first corresponds a certain locus in the second and vice versâ. We can say that the $x$ and $y$ groups correspond, and in fact the importance of the correspondence depends largely on these groups. For example, commencing with the ordinary projective group in space of three dimensions, we may take the equation $x_{3}=x_{1} y_{1}+x_{2} y_{2}+y_{3}$ as the equation system $\phi=0$. We thus establish a correspondence between points in the space $y$, and planes in the space $x$. Now planes in the space $x$ passing through a given line are transformed by the $x$ group into planes passing through another line. This condition must be an invariant one for points in the $y$ space. We see therefore that points $y$ lying on a line transform into points lying on a line. Also the cross ratio of four $x$ planes through a given line is invariant under all transformations of the $x$ group, and hence the cross ratio of four $y$ points lying on a line is invariant under all transformations of the $y$ group. The $y$ group is in fact also projective. This idea may be immediately applied to the general case, and we see that any relation among different $x$ loci gives an invariant relation among the corresponding $y$ points, and conversely. It is immediately obvious that the correspondence considered is a generalization of a contact transformation. The Lie transformation of straight 
lines into spheres gives a good idea of the importance of the underlying group. We mention two theorems.*

1. There are $\mathbf{1 5}$ infinitesimal contact transformations of spheres into spheres, and there are 15 infinitesimal transformations of straight lines into straight lines. Each of these sets forms a group and the two groups are simply isomorphic.

2. There are 10 infinitesimal point transformations of spheres, and there are ten infinitesimal transformations of straight lines into straight lines, which leave a given linear complex invariant. The two groups are again simply isomorphic.

\section{$\S 2$.}

We commence with the projective group in space. This group leaves invariant the family of all straight lines. We take for the equations $\phi=0$ the two:

$$
x_{1}=y_{1} x_{3}+y_{3}, \quad x_{2}=y_{2} x_{3}+y_{4} .
$$

This gives a correspondence between a manifold $x$ of three dimensions and one $y$ of four. To a line in the $x$ space corresponds a point in the $y$ space, and to a point in the $x$ space corresponds a $P_{2}$ of a particular type in the $y$ space. Corresponding to the projective group we have a 15 parameter group in four dimensions. The first preserves intersecting lines and hence there exists an invariant relation among two specially selected $y$ points. If two lines $y$ and $y^{\prime}$ intersect, then

$$
\left(y_{1}-y_{1}^{\prime}\right)\left(y_{4}-y_{4}^{\prime}\right)-\left(y_{2}-y_{2}^{\prime}\right)\left(y_{3}-y_{3}^{\prime}\right)=0 .
$$

Hence if we regard $y$ as fixed, all the points on a certain hypersurface of the second order must transform into points on a similar hypersurface. It is at once seen that the above relation may be made symmetrical by taking instead of the $y$ 's themselves certain linear functions of them, namely,

$$
y_{1}=Y_{1}+i Y_{2}, y_{4}=Y_{1}-i Y_{2}, y_{2}=-Y_{3}-i Y_{4}, y_{3}=-Y_{3}+i Y_{4} .
$$

We have now the relation between two points in the $Y$ space,

$$
\left(Y_{1}-Y_{1}^{\prime}\right)^{2}+\left(Y_{2}-Y_{2}^{\prime}\right)^{2}+\left(Y_{3}-Y_{3}^{\prime}\right)^{2}+\left(Y_{4}-Y_{4}^{\prime}\right)^{2}=0,
$$

invariant under the transformations of a 15 parameter group. The 15 parameter group must therefore transform all points lying on a line which meets the sphere at infinity into points on another such line. It must therefore leave this sphere invariant. It must leave invariant the relation

$$
d Y_{1}^{2}+d Y_{2}^{2}+d Y_{3}^{2}+d Y_{4}^{2}=0
$$

* See Lovert, Annali di Mathematica, ser. III', vol. 7 (1902), p. 39. 
and therefore it must be such that

$d Y_{1}^{\prime 2}+d Y_{2}^{\prime 2}+d Y_{3}^{\prime 2}+d Y_{4}^{\prime 2}=\rho\left(Y_{1} Y_{2} Y_{3} Y_{4}\right)\left(d Y_{1}^{2}+d Y_{2}^{2}+d Y_{3}^{2}+d Y_{4}^{2}\right)$,

where $Y_{1}^{\prime}, Y_{2}^{\prime}, Y_{3}^{\prime}, Y_{4}^{\prime}$ is the point obtained from $Y_{1}, Y_{2}, Y_{3}, Y_{4}$ by any operation of the group. The transformation must therefore be conformal. Conversely, if any infinitesimal conformal transformation be performed in the $\boldsymbol{Y}$ space it will give a transformation of lines into lines in the $x$ space and all lines through a given point will transform into lines through a point. It will therefore be a point transformation which preserves straight lines and will thus be projective. Hence we have the theorem:

The general projective group in three dimensions and the general conformal group in four are simply isomorphic.

Further, it is easily seen that a hypersurface of the second order contains an infinite number of straight lines of which $\infty^{1}$ go through any point on the surface. Conversely this property defines a hypersurface of the second order. Now a hypersphere is such a surface and the lines in question are all minimal lines. Hence a hypersphere must transform into a hypersurface of the second order under the general conformal group. But this group leaves the sphere at infinity invariant. Hence the transformed surface must be a hypersphere. We see therefore that there must exist some complex of lines in three dimensional space which is transformed into a similar complex by all projections. This complex is given by

$$
Y_{1}^{2}+Y_{2}^{2}+Y_{3}^{2}+Y_{4}^{2}+2 A Y_{1}+2 B Y_{2}+2 C Y_{3}+2 D Y_{4}+E=0,
$$

where $A, B, C, D, E$ are constants. This becomes

$$
\left(y_{1} y_{4}-y_{2} y_{3}\right)+a y_{1}+b y_{2}+c y_{3}+d y_{4}+e=0,
$$

where $a, b, c, d, e$ are constants. It is in fact a linear complex. Hence we see that projection changes a linear complex into a linear complex, and further, this is the most general point transformation that will do so. Incidentally we note that a correspondence is established between a linear complex and a hypersphere.*

Now two $S_{3}^{\prime}$ 's have one common invariant under all conformal transformations, namely, their angle of intersection. Hence there is one invariant under all projections for two linear complexes. We shall find it convenient to speak, instead of the linear complex, of the screw for which the lines of the linear complex are null lines. Then a screw corresponds to an $S_{3}$.

* Krein, Mathematische Annalen, vol. 5 (1872), p. 264. 
Let there be any wrench on a given screw, and consider the moment of the system round any line. If

$$
S \equiv Y_{1}^{2}+Y_{2}^{2}+Y_{3}^{2}+Y_{4}^{2}+\cdots \text { ete. }=0
$$

be the $S_{3}$ corresponding to the given line, then the moment in question is $\lambda S$, where $\lambda$ is defined as the "intensity" of the wrench. Now consider any two screws; associate intensity $\lambda /(\lambda+\mu)$ with the first, $\mu /(\lambda+\mu)$ with the second, and combine. We get a unit wrench on a third screw which lies on the cylindroid determined by the two original screws. We conclude that to a system of screws on a cylindroid corresponds a system of $S_{3}$ 's having a common sphere. Such a coaxial system includes two points $S_{3}$ 's. Hence there are two screws of zero pitch on a cylindroid. The two points thus determined are inverse points with respect to any oue of the linear system of $S_{3}$ 's. Now consider any $S_{3}$ containing both these points; it corresponds to a screw which has both these lines as null lines, and is therefore reciprocal to both the screws of zero pitch; hence it is reciprocal to all the screws of the cylindroid. But any $S_{3}$ through two inverse points of $S_{3}^{\prime}$ cuts $S_{3}^{\prime}$ orthogonally. Hence if the mutual invariant of two screws vanishes the two corresponding $S_{3}$ 's cut at right angles.

Now consider two $\boldsymbol{P}_{2}$ 's in the four dimensional space; if every line in one is perpendicular to every line in the other, the two $P_{2}$ 's are said to be at right angles. Take any $S_{3}$ passing through a fixed $S_{2}$ in a fixed $P_{2}$, and let $P_{3}$ contain this $P_{2}$. All $S_{3}$ 's with their centres in $P_{3}$ will have their centres on a fixed line perpendicular to the $P_{2}$ and passing through the centre of the fixed circle. Hence all $S_{3}$ 's through the fixed circle will have their centres in a fixed $P_{2}$ perpendicular to the $P_{2}$ of the circle, and passing through its centre (the two $P_{2}^{\prime}$ 's have of course only one point common). There will thus be a locus in the second $P_{2}$ corresponding to the point spheres of the system. Two points of this locus lie in any $P_{3}$ through the given $P_{2}$. These points lie on the line through the centre of the fixed circle perpendicular to the $P_{2}$. They are equidistant from the centre, and this distance is independent of the particular $P_{3}$ selected. Hence the locus of point spheres of the system is another circle; the two circles have the same centre, and it is easy to see that the sum of the squares of the radii is zero. The relation between the two circles is reciprocal. The whole system of $S_{3}$ 's is determined by any three independent ones (i. e., ones not having a common $S_{2}$ ). If we translate these results into three dimensions we see that given three screws there is a single infinity of lines of the zero pitch belonging to the system thus determined. There exists a single infinity of screws of zero pitch reciprocal to the whole system. To the one system corresponds a circle, and to the other another circle which is definite when the first is given. We thus see that to a regulus corresponds a circle, and to the lines meeting the rays of a regulus correspond the points of another circle. Similar considerations may 
be readily applied to the system arising from the four or five screws. Four $S_{3}$ 's have two points common, and the reciprocal system thus includes two point $S_{3}$ 's; this system is in fact coaxial. The five system consists of $S_{3}$ 's orthogonal to a given $S_{3}$. Now suppose we fix a given screw; by an inversion we may make the corresponding $S_{3}$ a $P_{3}$, and then a correspondence is established between the lines of a given complex and points of a $P_{3}$; for any point in the $P_{3}$ corresponds to a screw of zero pitch reciprocal to the given screw, that is to say, any point in the $P_{3}$ corresponds to a line of the complex determined by the fixed screw. Any $S_{3}$ meets the $P_{3}$ in a sphere, and for a given sphere there is a single infinity of $S_{3}$ 's. This single infinity is determined by any given $S_{3}$ and the $P_{3}$. It follows that we may regard a sphere as corresponding to all the screws of a cylindroid which contains the $P_{3}$. Now a cylindroid is determined uniquely by two of its screws, hence the $P_{3}$ and any line determine a unique sphere. But corresponding to any sphere there are two point $S_{3}$ 's, that is to say two lines, and hence the correspondence between lines and spheres is a 2-1 correspondence. The points are images in the $P_{3}$, and therefore the lines are conjugates with respect to the given complex. Now consider any two spheres. They are the intersections of the $P_{3}$ with two point $S_{3}^{\prime}$ 's; the two spheres intersect in a circle, and therefore the two point $S_{3}^{\prime}$ 's lie on a circle which is perfectly determined if the first circle is given. The rays of a regulus correspond to the circle thus determined. The regulus is not general, for it is self conjugate with respect to the fundamental screw, and the directors are therefore null lines of this screw. Suppose that the two spheres touch; both circles now become point circles, and hence each of them is a pair of minimal lines. Hence if two spheres touch, the four corresponding four dimensional points lie on two minimal lines. As the spheres are supposed general, no two points corresponding to the same sphere lie on the same minimal line, and therefore the points lie by pairs on two minimal lines. Hence the corresponding lines in the three dimensional space intersect in pairs. $\mathrm{W}^{\top}$ e note that this also includes the theorem that if two straight lines intersect their conjugates intersect. This correspondence between lines and spheres is of course that of LiE. Without inversion we should have a correspondence between lines in ordinary space and spheres in elliptic space.

We may take six $S_{3}$ 's as coördinates, and thus express any point by means of its powers from these $S_{3}^{\prime}$ 's. The coördinates thus used are the equivalent in four dimensions of DARBoux's* pentaspherical coördinates in three. As a particular simplification we take the $S_{3}^{\prime}$ 's to be mutually orthogonal, and we thus see that the method of discussing screws by referring them to six co-reciprocal coördinate screws is strictly in correspondence with DARBoux's sphere geometry.

* See DaRboux, Théorie des surfaces, vol. 1, p. 213. 
We take the powers mentioned to be $z_{1}, z_{2}, z_{3}, z_{4}, z_{5}, z_{6}$, and then any $S_{3}$ is given by a linear relation among the $z$ 's. The condition for orthogonality of two $S_{3}^{\prime}$ s

and

$$
\lambda_{1} z_{1}+\lambda_{2} z_{2}+\lambda_{3} z_{3}+\lambda_{4} z_{4}+\lambda_{5} z_{5}+\lambda_{6} z_{6}=0
$$

is

$$
\lambda_{1}^{\prime} z_{1}+\lambda_{2}^{\prime} z_{2}+\lambda_{3}^{\prime} z_{3}+\lambda_{4}^{\prime} z_{4}+\lambda_{5}^{\prime} z_{5}+\lambda_{6}^{\prime} z_{6}=0
$$

$$
\lambda_{1} \lambda_{1}^{\prime}+\lambda_{2} \lambda_{2}^{\prime}+\lambda_{3} \lambda_{3}^{\prime}+\lambda_{4} \lambda_{4}^{\prime}+\lambda_{5} \lambda_{5}^{\prime}+\lambda_{6} \lambda_{6}^{\prime}=0 \text {. }
$$

Hence if an $\mathrm{S}_{3}$ cuts itself orthogonally,

$$
\lambda_{1}^{2}+\lambda_{2}^{2}+\lambda_{3}^{2}+\lambda_{4}^{2}+\lambda_{5}^{2}+\lambda_{6}^{2}=0 .
$$

This is therefore the condition for a point $S_{3}$. If the point $S_{3}$ cuts

orthogonally,

$$
\overline{S_{3}} \equiv \sum_{r=1}^{6} \lambda_{r}^{\prime} z_{r}=0
$$

$$
\lambda_{1} \lambda_{1}^{\prime}+\lambda_{2} \lambda_{2}^{\prime}+\lambda_{3} \lambda_{3}^{\prime}+\lambda_{4} \lambda_{4}^{\prime}+\lambda_{5} \lambda_{3}^{\prime}+\lambda_{6} \lambda_{6}^{\prime}=0 .
$$

Hence the point $\lambda_{1}, \lambda_{2}, \ldots, \lambda_{6}$ lies on $\bar{S}_{3}$, and this point is therefore the centre of the point $S_{3}$. Hence among the six coördinates of any point there exists the relation

$$
\sum_{r=1}^{6} z_{r}^{2}=0 . \dagger
$$

Consider the general relation

$$
\text { §3. }
$$

$$
Y_{1}^{2}+Y_{2}^{2}+Y_{3}^{2}+Y_{4}^{2}+2 X_{1} Y_{1}+2 X_{2} Y_{2}+2 X_{3} Y_{3}+2 X_{4} Y_{4}+X_{5}=0
$$

in conjunction with the conformal group in the space $Y$. This equation is invariantive, and hence it establishes a correspondence between space of four and that of five dimensions. The group for the $X$ 's changes $P_{4}^{\prime}$ 's of a particu-

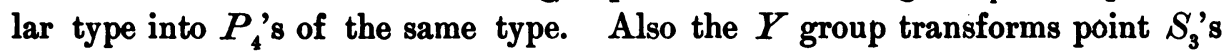
into point $S_{3}^{\prime}$ 's, and therefore the $X$ group must leave invariant the manifold

$$
X_{1}^{2}+X_{2}^{2}+X_{3}^{2}+X_{4}^{2}=X_{5} \text {. }
$$

Coaxial $S_{3}^{\prime}$ 's in the $Y$ space correspond to lines in the $X$ space, and thus lines are transformed into lines in the $X$ space. The $X$ group is thus seen to be projective and to keep a particular quadric invariant. Hence the projective group in five dimensions which preserves a particular quadric is isomorphic to the general projective group in three dimensions and to the

† In connection with this $S_{3}$ geometry see DarBoux, loc. cit. 
general conformal group in four. To a general line corresponds a coaxial system of $S_{3}$ 's, or, since the system is determined by its common sphere, the correspondence is one between lines in five and spheres in four dimensions. To a system of lines having a common point corresponds a system of spheres lying on a common $S_{3}$. To the points of a general $P_{2}$ correspond $S_{3}^{\prime}$ 's passing through a given circle, and hence we may say that to a given $P_{2}$ in five dimensions corresponds a circle in four. If two points are conjugate with respect to the quadric, the corresponding $S_{3}^{\prime}$ 's cut orthogonally. Hence to the points of a $P_{4}$ correspond all the $S_{3}^{\prime}$ 's cutting a given $S_{3}$ orthogonally. To the points of a $P_{3}$ correspond the $S_{3}^{\prime}$ 's orthogonal to two given $S_{3}^{\prime}$ 's, or passing through two given points, and so on. Comparing the five dimensionality with the three dimensionality with which we started, we see that, e. g., to straight lines correspord points on the fundamental quadric, and inversion with respect to a linear complex is equivalent to reflection of the quadric in a given point.

It is convenient to project the fundamental quadric into an $S_{4}$. This may be done by taking new coördinates $\xi_{1}, \xi_{2}, \xi_{3}, \xi_{4}, \xi_{5}, \xi_{6}$, where

$$
\rho x_{1}=\xi_{1}, \rho x_{2}=\xi_{2}, \rho x_{3}=\xi_{3}, \rho x_{4}=\xi_{4}, \rho x_{5}=-\left(\xi_{5}+1\right), \rho=\left(\xi_{5}-1\right) .
$$

The quadric now becomes

$$
\sum_{r=1}^{6} \xi_{r}^{2}=1
$$

and the $S_{3}$ in the four dimensional space becomes

$$
z_{6}=\xi_{1} z_{1}+\xi_{2} z_{2}+\xi_{3} z_{3}+\xi_{4} z_{4}+\xi_{5} z_{3},
$$

where the $z$ 's are a mutually orthogonal set of $S$ 's.

The plane representation for the three system of screws due to Sir RoBerT BALL * is seen to be a particular case of this correspondence; and we note that the projective group in space which projects a three system into itself is simply isomorphic with the plane projective group that preserves a given circle.

We can at once give a geometrical interpretation in five dimensions to any screw system. To a 2-system corresponds the system of points on a line, and in general to an $n$ system corresponds the points of a $P_{n-1}$. The most general projection of an $n$ system into itself corresponds to the most general projection of a $P_{n-1}$ into itself which preserves a given $S_{n-2}$. For example, the most general projection of a cylindroid into itself corresponds to the homographic transformation of a straight line which preserves two given points and so on.

We note that corresponding to a $P_{n}(n=0, \ldots, 4)$, in the five dimensionality there is a reciprocal $P_{4-n}$ with respect to the fundamental quadric. Hence an $n$ system of screws has a $6-n$ system of reciprocal screws.

* Theory of Screws (1900). chap. 15. 
Now consider $n$ screws and let there be associated with them intensities $\lambda_{1}, \cdots, \lambda_{n}$. The resultant wrench will be of intensity $\lambda_{1}+\cdots+\lambda_{n}$, and if $\xi_{r 1}, \cdots, \xi_{r 5}$ are the coördinates of the point corresponding to the $r$ th screw, the point corresponding to the resultant screw has the coördinates

$$
\sum_{s=1}^{n} \xi_{s t} \lambda_{s} / \sum_{s=1}^{n} \lambda_{s} \quad(t=1, \cdots, 5) .
$$

Hence finding the resultant wrench is equivalent to finding the mass centre of points $\xi$ with associated multiples $\lambda$. The conditions for possibility of equilibrium of wrenches on $n$ given screws follow at once from the five dimensional representation. In fact the corresponding points must obviously lie in a $P_{n-1}$. Hence the $n$ screws must belong to an $n-1$ system.

BRYN MAWR, January, 1906. 